\title{
Berichtigungen und Zusätze zu Band LXXIV.
}

p. 3 Z. 10 v. 0. statt Ostpersiens lies Westpersiens

p. 3 Z. 17 v. 0. " Garbad " Sarhad und später

p. 6 Z. 4 v. 0. "Samehol "Samchol

p. 6 Z. 8 v. u. "Mudschaabad " Mudschnabad

p. 7 Z. 9 v. 0. "Nalsrich "Nalsrieb und spăter

p. 9 Z. 10 จ. a. " Hussem-abad " Hussein-abad

p. 9 Z. 6 v. u. " Gizkuh " Sirkub

p. 10 Z. 7 v. o. " Moschnil-Ebene lies Maschkil-Ebene

p. 11 Z. 19 จ. o. " Dschaudak lies Dscbandak

p. 13 Z. 12 v. u. " Torach " Torasch

p. $15 \mathrm{Z} 12$ v. 0 . statt $20 \sigma^{x} \sigma^{x}$ lies $26 \sigma^{x} \sigma^{x}$

p. 17 Z. 16 จ. u. " Kutsehé " Kutsché

p. 23 Z. 5 v. u. " Kunscha " Kuuscha und spater

p. 27 Z. 11 V. o. "Sinon "Sinou

p. 28 Z. 1 v. u. " Schaschgirt-Cala lies Schaschgirt-bala.

p. 29 Z. 5 v. u. "Dchorn lies Dschoru und spáter

p. $30 \mathrm{Z} 8$ จ. o. " Bola-Chaf lies Bala-Chaf.

p. 32 Z. 18 v. 0. " Tebbas "Tebbes

p. 34 Z. 12 จ. o. "Leinelabad" Seinelabad

p. 35 Z. 6 v. 0. " Demarend " Demavend

p. 38 Z. 3 v. o. " Sija-Maussur lies Sija-Manssur

p. 43 Z. 20 v. 0. " Hurmun lies Hurmuk

p. 44 Z. 8 จ. 0. " Tschagastagan lies Tschagartagau

p. 44 7. 3 v. 0. " Cala lies bala

p. 46 Z. 12 v. u. "Machemik lies Machunik.

p. 51 Z. 7 v. u. "Zugvögel " Jungrögel

p. 51 Z. 2 v. u. "Sennatscha " Seimatscha

p. 97 Z. 11 v. o. " wollen " sollen

p. 98 Z. 15 v. 0 . " doch " dort

p. 98 Z. 7 จ. u. "Zinden " Zinder

p. 99 Z. 16 จ. u. " aber " also

p. $101 \mathrm{Z} .10$ v. o. "Marchen " Maschen

p. 104 Z. 6 v. o. "seine " eine

p. 104 Z. 8 v. 0. " Emudi " Ennudi

p. $104 \mathrm{Z}, 18-19$ v. 0 . statt urasiatischer lies eurasiatischer

p. 105 Z. 13 v. 0 . statt dennoch lies demnach

p. $132 \mathrm{Z} .2$ v. o. dsjorsn(u)ied ist zu streichen

p. 132 Z. 8 v. o. die Silbe si und die folgenden Punkte sind so hoch zu stellen wie der letzte Punkt.

p. 135 das Notenbild gebort zum Waldschwirrvogel, nicht zum Girlitz. 
p. 370 Fig. 1. Hilfsbeobaehtungsstellen der Vogelwarte Helgoland im Jahre 1925: Langeoog, Mellum und Cuxhafen. Die Errichtung weiterer Stationen (punktierte Kreise) wird erstrebt.

Gegentiber p. 370 Fig. 2. Lerchenzug im Januar 1924 auf Helgoland und das Wetter auf Helgoland (-), boi Kopenhagen (-.) und bei Helder $(\cdots)$.

p. 372 Fig. 3. Das bauptsåchlichsto Wandergebiet der Helgolander Zugvogel. - Diese Zeichnung dient als Hilfsmittel bei Untersuchungen über den Zusammenhang $z$ wischen Zug and Witterung (nảheres im Text).

p. 374 Fig. 4. Schematische Darstellung der Vogelfangreusen der Vogelwarte Helgoland. Links altes (1922), rechts neues Modell (1925). $\mathrm{T}=$ Tür, $\mathrm{Kl} .=$ Klappe, $\mathrm{F}=$ Falltür, $\mathrm{K}=\mathrm{Kafig}$.

p. 496 Z. 29-19 v. u. statt Motacilla alba aralensis lies Motacilla alba uralensis.

p. 585 Z. 16 v. 0 . statt 1793 lies 1783 . 


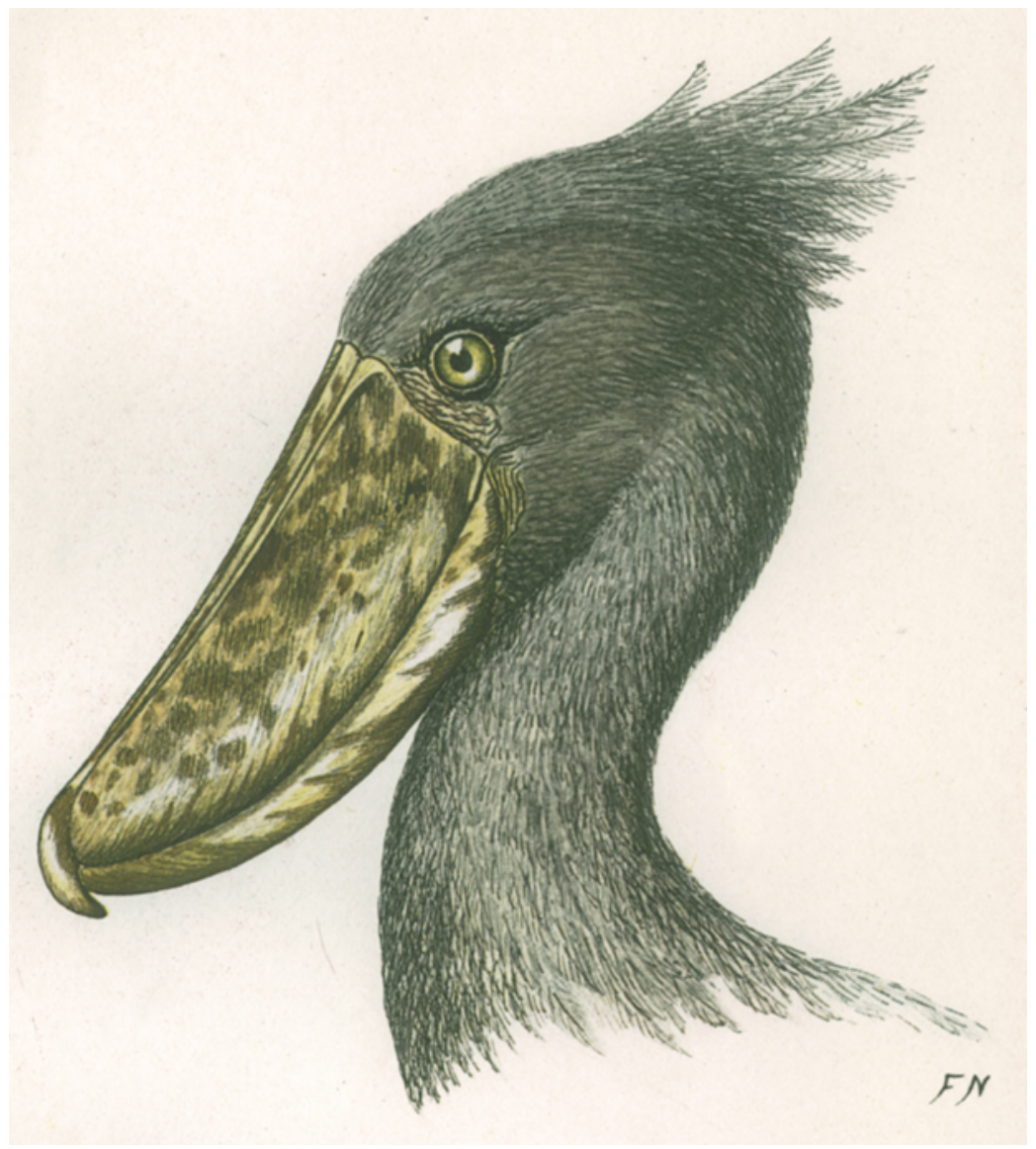

Balaeniceps rex, Gould 
$\frac{\bar{\Xi}}{\stackrel{\Xi}{0}}$
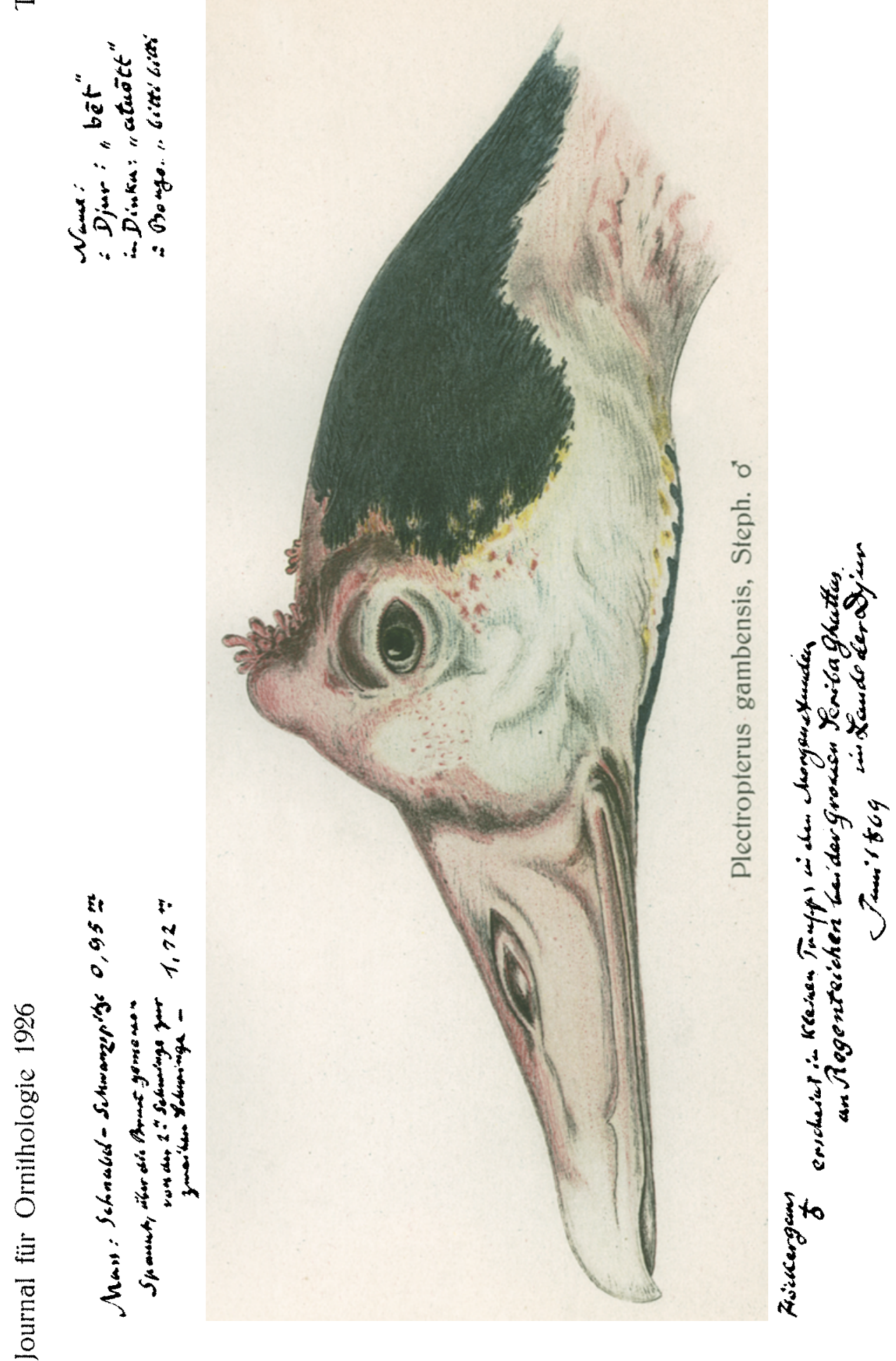

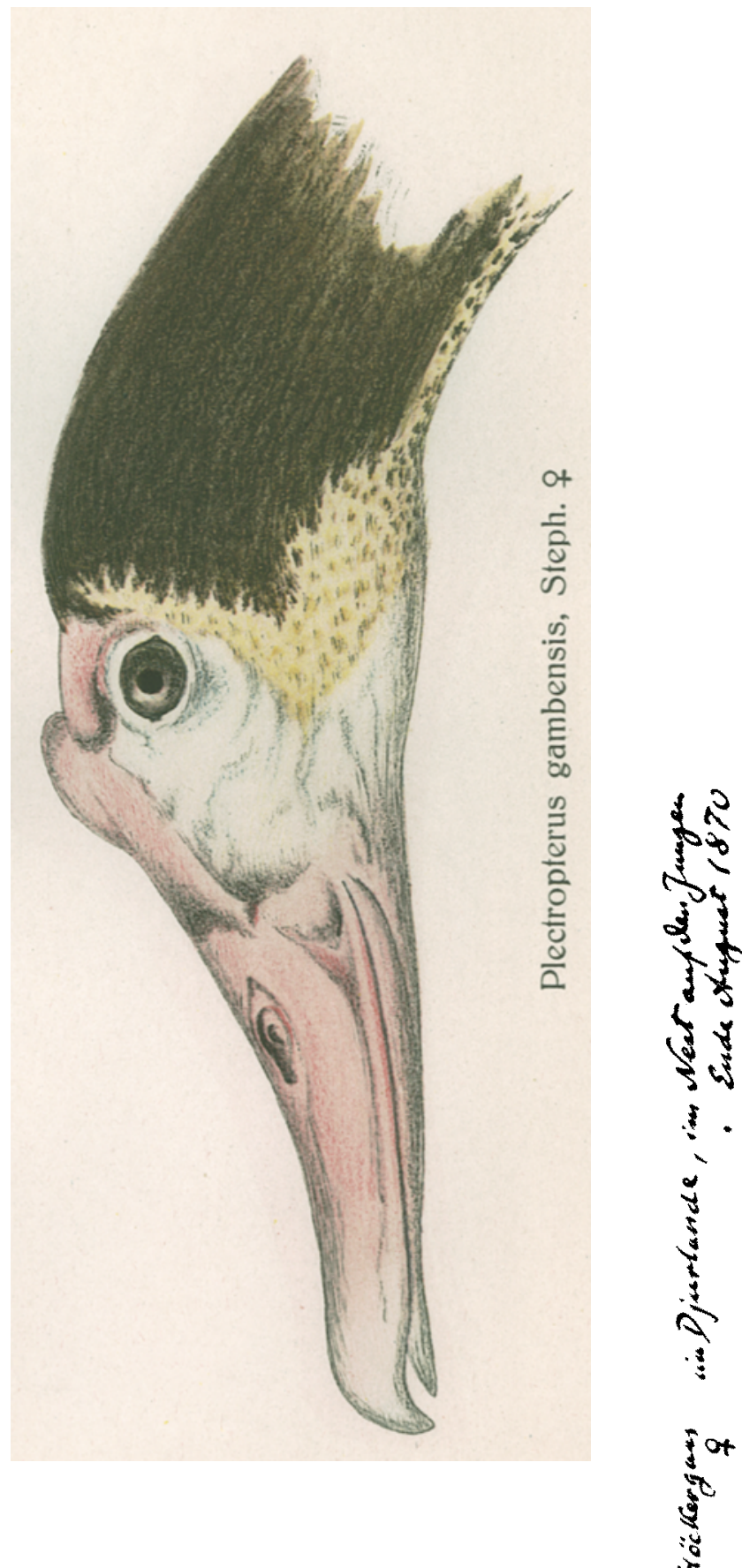


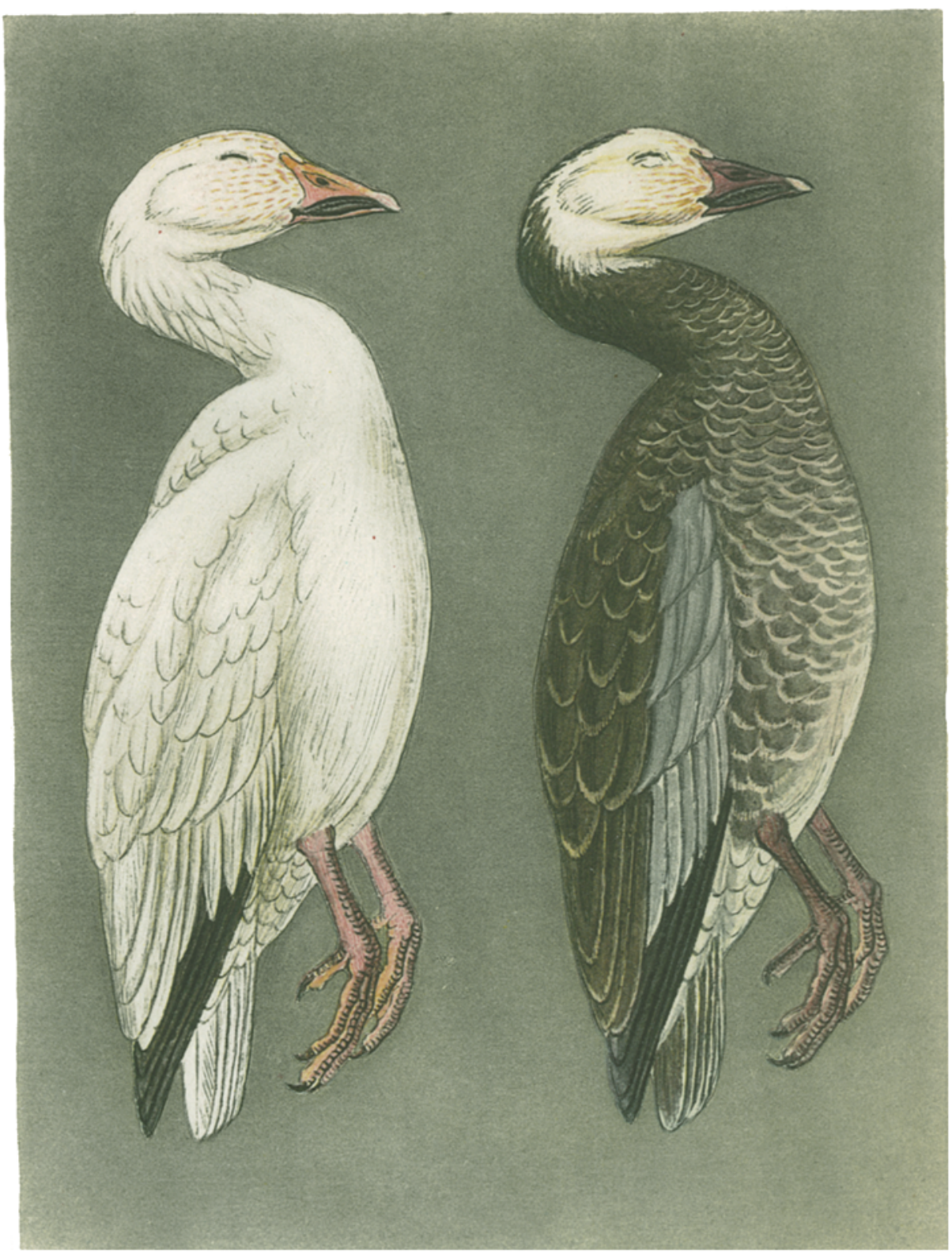

Anser caerulescens (1..)

Mutanse

„Anser hyperboreus (Pall.)"
Urspriingliche Phase

Anser caerulescens (L.) 


\section{Erklärung zu Tafel V.}

1. Chlorophoneus multicolor (Gray) $\sigma^{x}-1 \mathrm{~m}$. dessen Mutante:Chlorophoneus nigrithorax (Sharpe) $\sigma^{\pi}$ [Togo].

2. Lanius schach schach (L.) - 2 m. dessen Mutante: Lanius fuscatus L9ss. [Süd-China].

3-4. Sylvia atricapilla obscura Tschusi

3. ursprüngliche Phase, $\sigma^{\top}-3 \mathrm{~m}$. deren Mutante: Sylvia heineken Jard. of [Madeira].

4. ursprüngliche Phase, $\subsetneq-4 \mathrm{~m}$. deren Mutante: Sylvia heineken: Jard. $\%$ [Madeira]. 


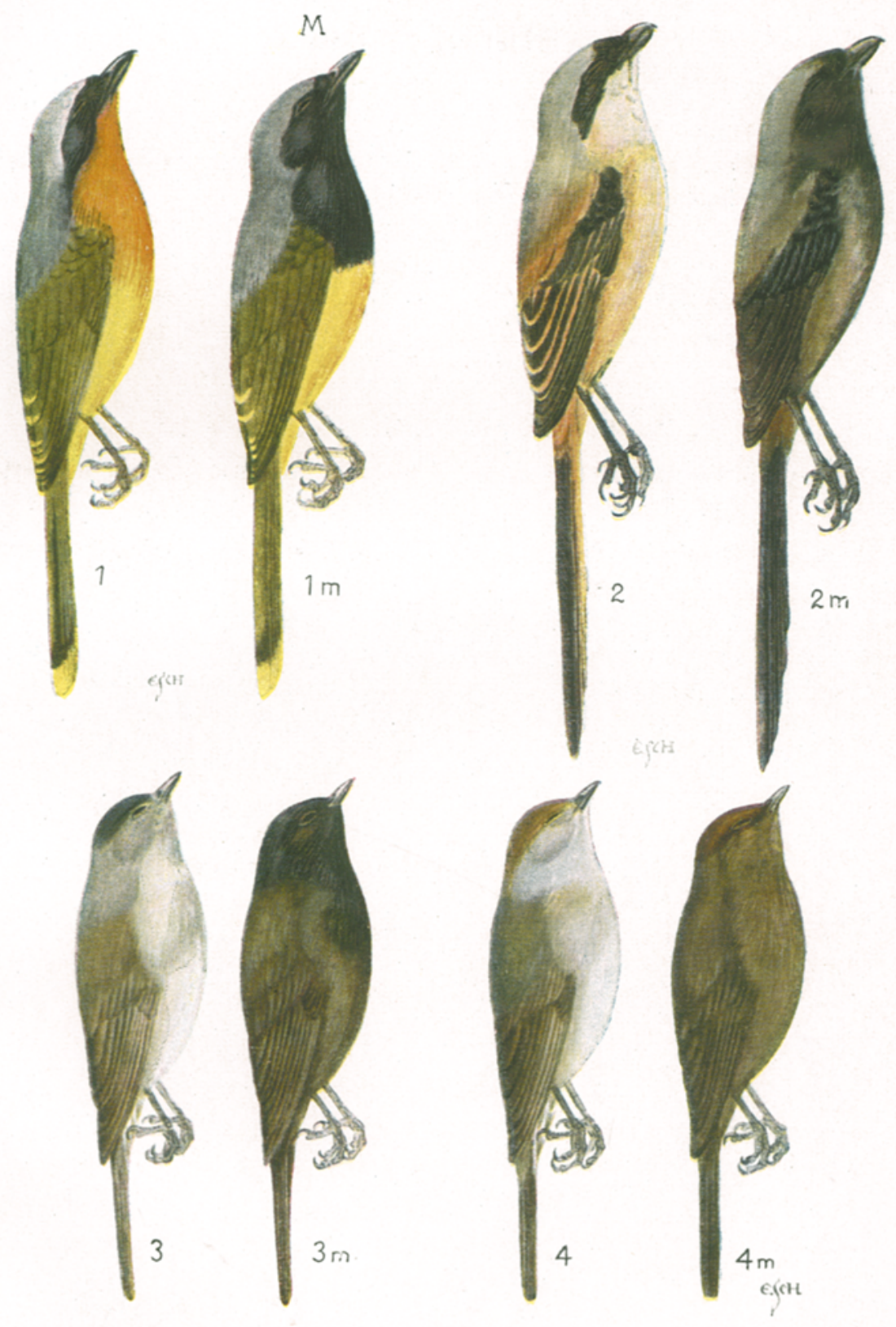




\section{Erklärung zu Tafel Vl.}

5-6. Charmosyna stellae goliathina R. \& H.

5. ursprüngliche Phase, $O^{x}-5 \mathrm{~m}$. deren Mutante: Charmosyna atrata Rothsch. $\sigma^{\top}$ [Deutsch-Neuguinea: Schraderberg].

6. ursprüngliche Phase, $\subsetneq-6 \mathrm{~m}$. deren Mutante: Charmosyna atrata Rothsch. $\&$ [Deutsch-Neuguinea: Schraderberg].

$Z$ u beachten : roter Bürzel des ơ bleibt ungeschwärzt, gelber Bürzel des $ᄋ$ verändert sich durch Einlagerung schwarzen Melanins zu Grün.

7-8. Poëphila gouldiae (Gould)

7. ursprüngliche Phase, $\sigma^{\Upsilon}-7 \mathrm{~m}$. deren Mutante: Poëphila mirabilis Des Murs ơ [Nordaustralien].

8. ursprüngliche Phase, $\subsetneq-8 \mathrm{~m}$. deren Mutante: Poëphila mirabilis Des Murs $\subsetneq$ [Nordaustralien].

$\mathrm{Zu}$ be a chte $\mathrm{n}$ : Die Mutante ist stets heterozygot veranlagt; die rote Gesichtsmaske ist beim of vollkommen, beim $\varnothing$ unvollkommen dominant über die schwarze Gesichtsmaske. 

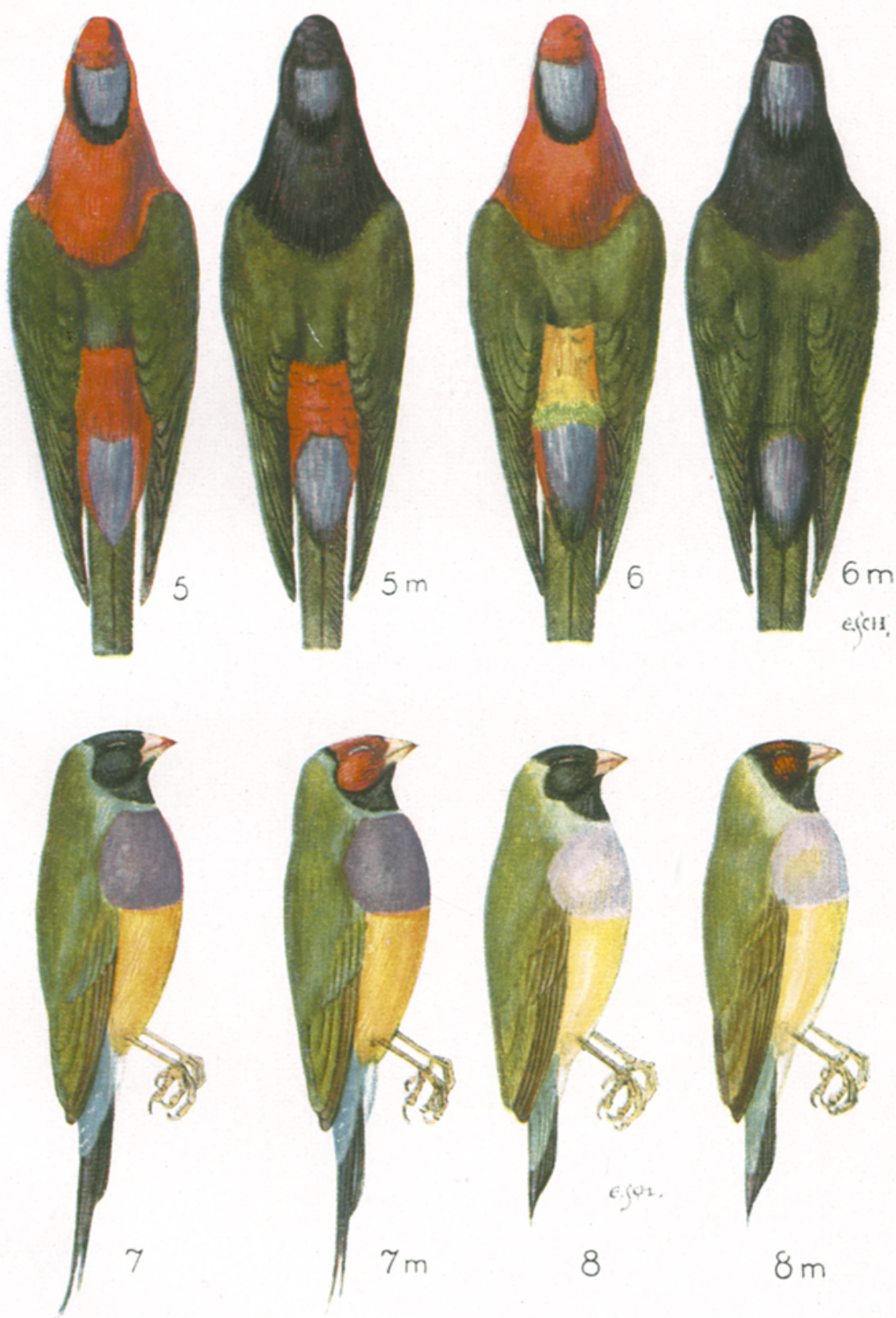


\section{Erklärung zu Tafel VII.}

9-10. Pavo cristatus L.

9. ursprüngliche Phase, $\sigma^{\top}-9 \mathrm{~m}$. deren rezessive Mutante: Pavo nigripennis Sclater $\sigma^{\top}$ [Domestikation in Europa].

10. ursprüngliche Phase, $\&-10 \mathrm{~m}$. deren rezessive Mutante: Pavo nigripennis Sclater $Q$ [Domestikation in Europa].

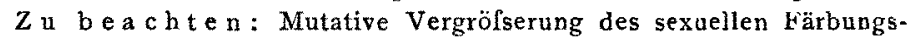
abstandes. Beide Geschlechter der Mutante sind im Dunenkleid weils.

11. Rhipidura flabellifera (Gmelin) - $11 \mathrm{~m}$. deren Mutante: Rhipidura fuliginosa (Sparrm.) [Neuseeland].

12. Khipidura albiscapa Gould [Südaustralien].

$\mathrm{Z}$ u bea chten: Rh. flabellifera mit weifsem Brauenstreif, $R h$. fuliginosa mit weilsenı Schläfenstreif, Rh. albiscapa mit weifsem Schläfenund Brauenstreif.

13-14. Terpsiphone mutata L.

13. einzige Tracht des $Q-14$ ursprüngliche Phase des $\sigma^{\top}$ ad. - $14 \mathrm{~m}$. Mutante des $\sigma^{\top}$ ad. [Madagaskar] 

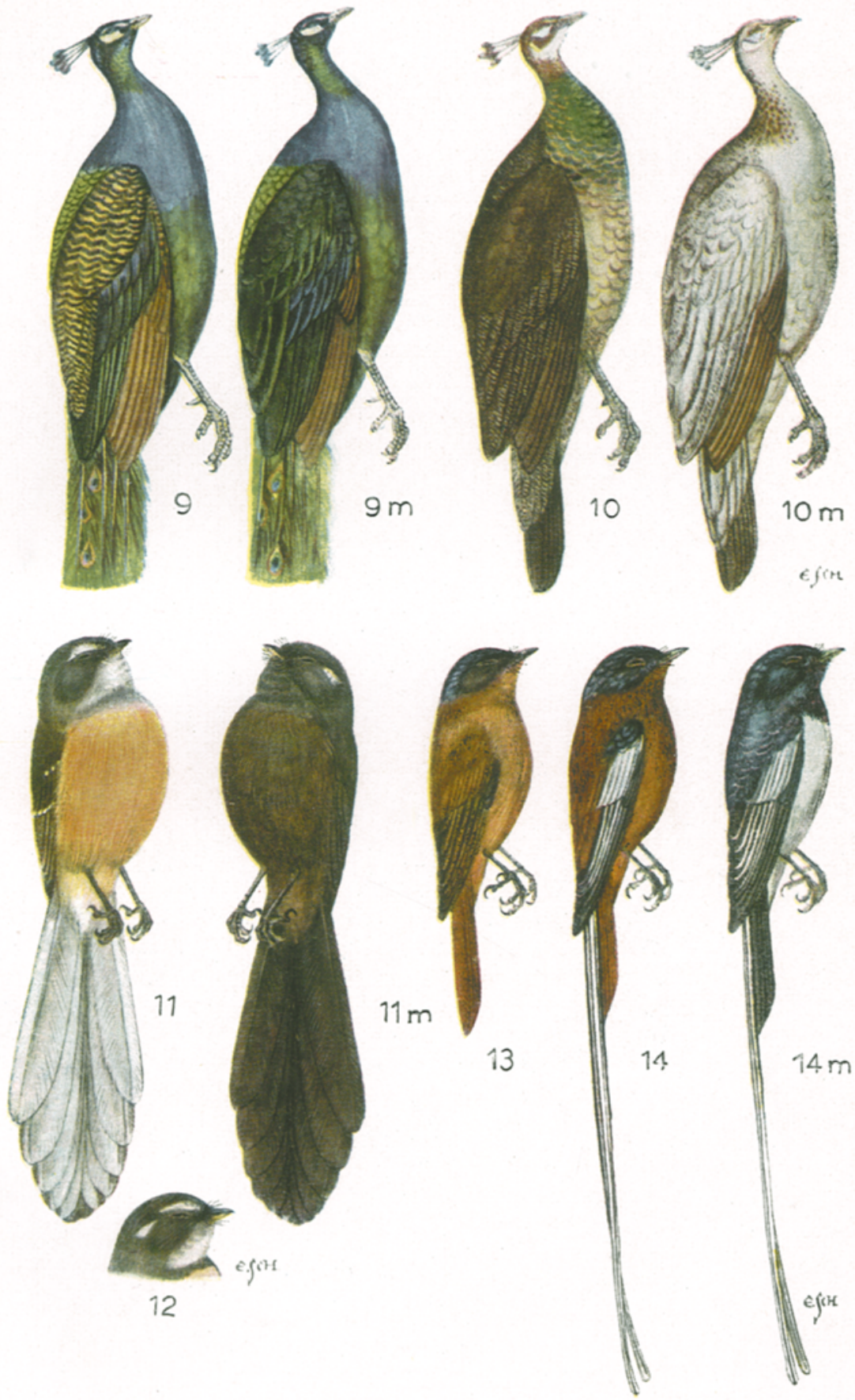


\section{Erklärung zu Tafel VIII.}

15. Accipiter novaehollandiae etorques (Salvad) - $15 \mathrm{~m}$. dessen Iutante: Accipiter novaehollandiae leucosomus (Sharpe) [Neuguinca].

16. Micronisus gabar (Daudin) - $16 \mathrm{~m}$. dessen Mutante: Micronisus niger (Bonn. \& Vieill.) [A frika].

$\mathrm{Z}$ u beachteen: Die weilse Bänderung des Schwanzes und der Scliwingen ist bei der Mutante exhalten geblieben und hat am Flügel sogar an Ausdehnung zugenommen, dabei die Regelmäfsigleit ihrer Anordnung einbüfsend.

17. Florida caerulea (L.) - $17 \mathrm{~m}$. dessen Mutante [Mittelamerika].

1․ Clamator jacobinus melanoleucus (Latb.) - $18 \mathrm{~m}$. dessen Nlutante: Clamator serratus (Sparrin.) [Südafrika].

$Z_{u}$ beachten: Der weilse Fliggelspiegel ist bei der Mutante erhalten geblieben. 

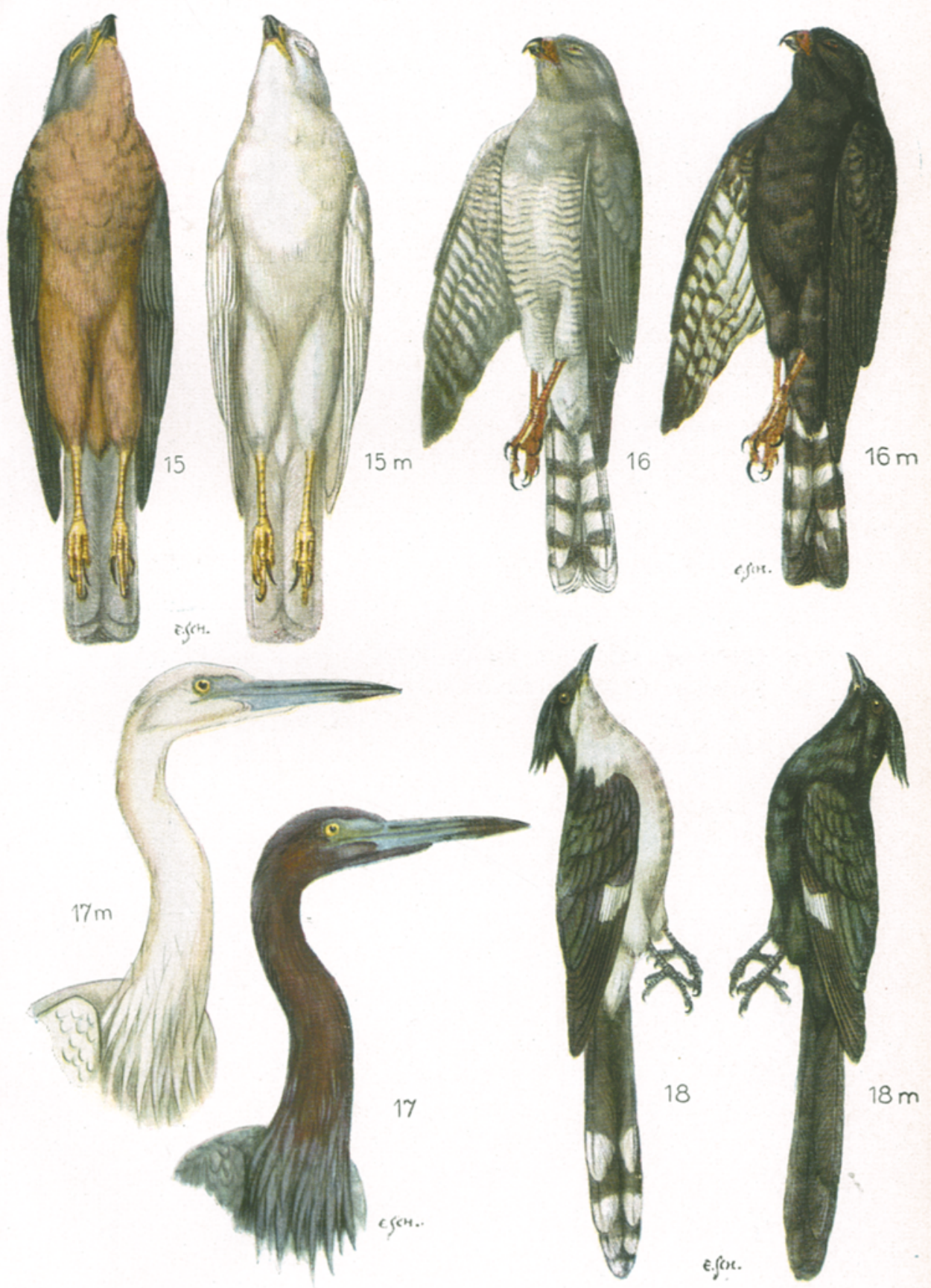


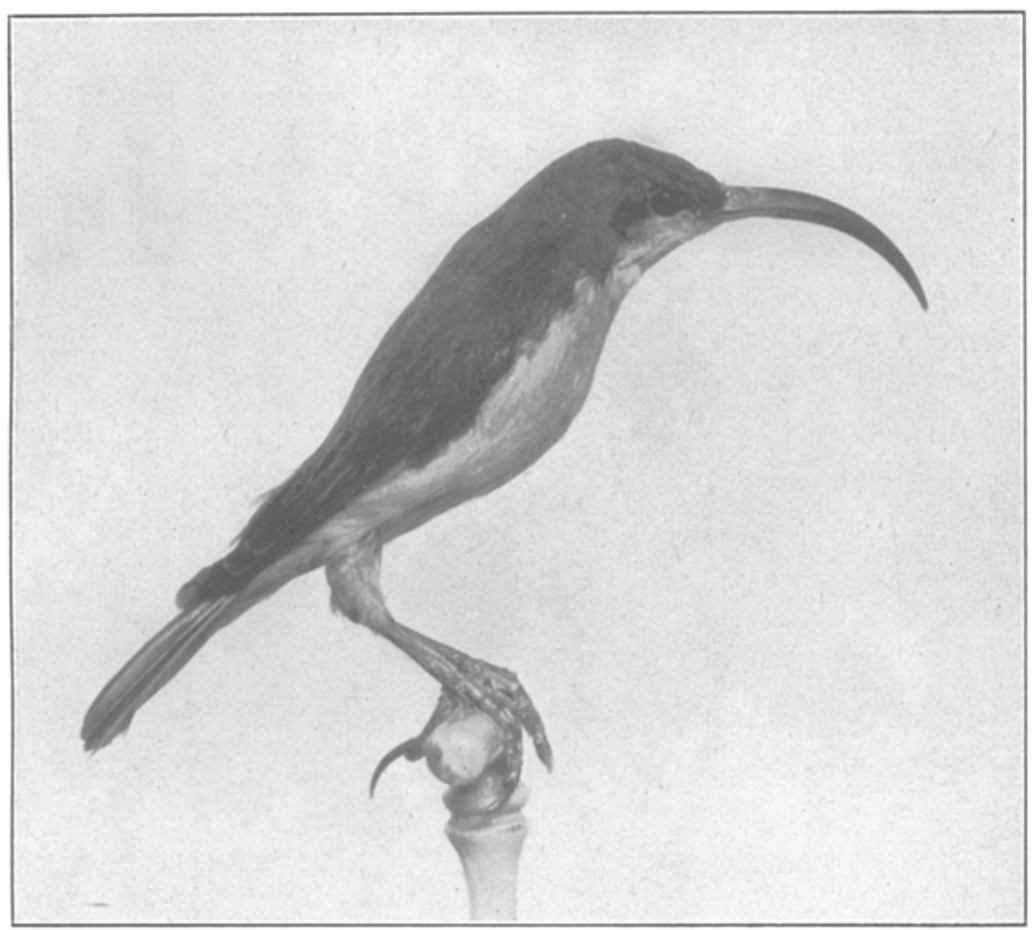

Sassius simplex Rothsch. u. Hart. 

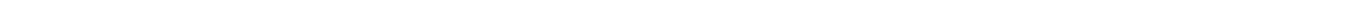


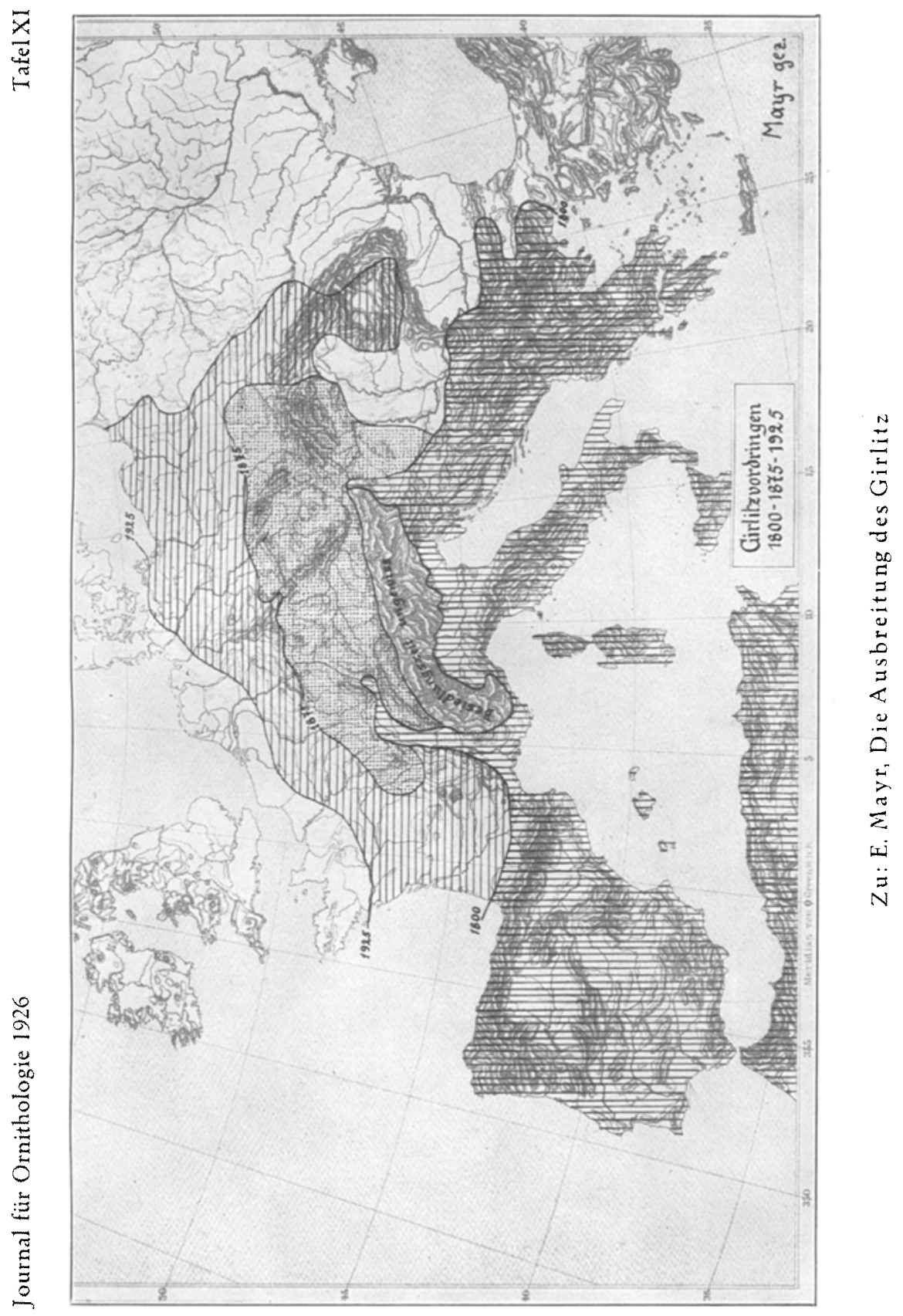




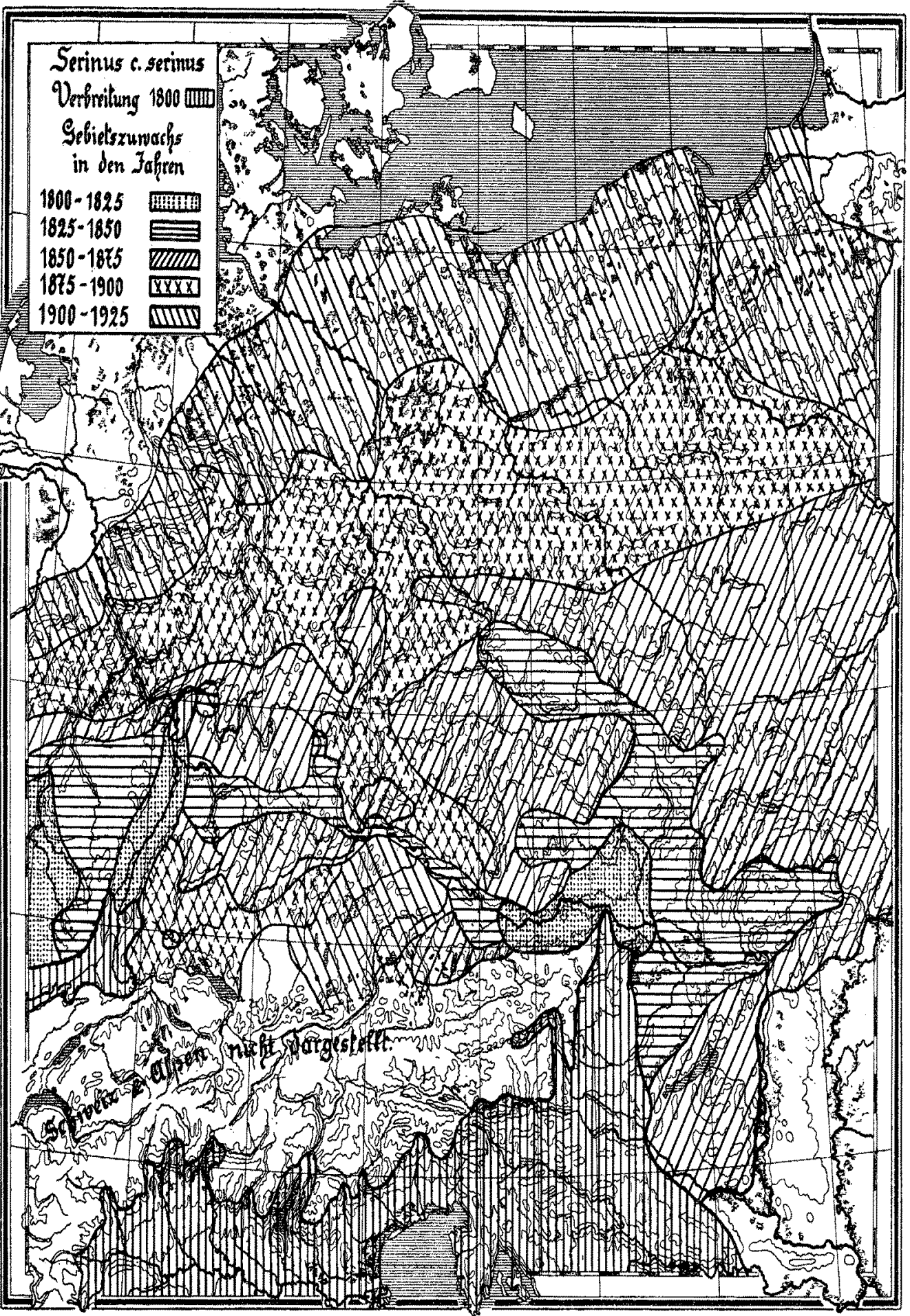

